\title{
Simultaneous blind gamma estimation
}

\author{
Javier Vazquez-Corral and Marcelo Bertalmío
}

\begin{abstract}
Blind gamma estimation is the problem of estimating the gamma function that is applied to a linear image both for perceptual reasons and for the compensation of the non-linear behavior of displays. Gamma values change both inter- and intracamera. In the latter case, the change comes from the use of different scene settings. In this paper we propose a new approach that relies on the use of more than a single image from the same scene. We estimate the gammas for all the different images at the same time with a method based on exploiting the structure of the standard in-camera processing pipeline. Our results improve over the state-of-the-art.
\end{abstract}

Index Terms-Blind gamma estimation, image enhancement

\section{INTRODUCTION}

Digital cameras process visual information in a non-linear manner. The main stages of the color processing pipeline can be summarized as follows [1], [2]

$$
\left[\begin{array}{l}
R \\
G \\
B
\end{array}\right]_{\text {out }}=\left(A \cdot\left[\begin{array}{l}
R \\
G \\
B
\end{array}\right]_{\text {in }}\right)^{\frac{1}{\gamma}}
$$

where $A$ is a $3 \times 3$ matrix comprising white balance and color encoding, $R G B_{i n}$ is the camera raw triplet at a given pixel location, and a pixel-based non-linear function defined as a power law of exponent $\frac{1}{\gamma}$, called gamma-correction, is applied to each pixel value [1], [3]-[6].

Eq. 1 tells us that to perform any image processing task on linear sensor values, the non-linear function should be first undone. This problem is known as blind gamma estimation and it is well-known in many different tasks, e.g. color constancy [7], [8] or camera shake removal [9].

A problem related to blind gamma estimation is camera response function (CRF) estimation. The main difference is that CRF estimation aims to obtain physical reliable values, therefore considering other non-linearities processed by the camera, such as gamut mapping. This premise deviates CRF estimation from our problem as the non-linearity is different for each of the three color channels, and converts the problem in a scene-dependent one: for a fixed value $R$ a different gamut mapping is applied depending on the $G$ and $B$ values. Nonetheless, we review CRF estimation works in the next section and compare our results versus a well-known one [10].

This paper presents a novel, accurate, and practical method for blind gamma estimation by exploiting the information given by the pipeline of Eq. 1 when different images of the same scene are available. To the best of our knowledge, this is the first work to address blind gamma estimation with several

Authors are with the Departament de Tecnologies de la Informació i les Comunicacions, Universitat Pompeu Fabra, Barcelona, Spain, \{javier.vazquez,marcelo.bertalmio\}@upf.edu. This work was supported by the European Research Council, Starting Grant ref. 306337, by the Spanish government, grant ref. TIN2012-38112, and by the Icrea Academia Award. images, therefore opening the door for further works in this direction. Our method does not require neither any knowledge about the cameras used (model, settings, etc.), nor a geometric calibration of the views, in contrary to [11] or [12], and it can be used under large variations of point of view and illumination. Our method is useful under different scenarios. Let us suppose we have a camera whose output we know we will want in linear form. In this case three options are possible. First, we can store the full RAW image; however, this approach requires a significant amount of memory space and therefore does not allow to take a large number of images. Also, RAW images require the user to specifically perform a number of essential operations (like demosaicing, conversion to a standard color space, color correction) which are automatically handled by the camera when the output image is in gamma-corrected, non-linear form. A second approach would be to color calibrate the camera, which allows to linearize a non-linear output. This process is time-consuming and expensive, requiring high-level technology unavailable to most users. Finally, another possibility is the use of blind gamma estimation methods. In this work we focus on this third approach and show that, if we know beforehand that we want our images to be linearized later (which is crucial for some applications such as illuminant estimation), we just need to take two or more versions of each photo, with different settings, and use our proposed method to estimate the gamma value for each one afterwards. Furthermore, even in the case that we only have one image, we just need to find another picture sharing part of the scene content (e.g. by searching on internet for another view of the same location): in this case, our method will also work as it does not require any knowledge about the camera.

We point out that this work extends an earlier idea presented in [13], where the computation of gamma ratios between two images was proposed as a pre-processing step for a color stabilization algorithm. Our contributions in this paper are twofold. First, we extend the blind gamma estimation process to more than two images. Second, we present an extensive set of experiments to demonstrate the adequacy of our method.

\section{RELATED WORK}

Farid [14] proposed the first major work on blind gamma estimation. He noticed that gamma correction introduces higherorder correlations in the frequency domain, and therefore he looked for the value of $\gamma$ where these correlations were minimized. Stamm and Liu [15] proposed a method for detecting global contrast enhancement by seeking out unique artifacts in the image histogram. Cao et al. [16] estimated $\gamma$ from the fact that gamma correction creates peaks in the histogram for low intensity values and gaps for high intensity values. 
In terms of CRF estimation from a single image, Lin et al. [10] focused on the color changes at the edges between objects in order to look for the CRF. The same authors adapted their work to grey-scale images obtained from an RGB original image [17]. $\mathrm{Ng}$ et al. [18] proposed the use of geometry invariants to select locally planar irradiance points. Tai et al. [19] presented a method for obtaining CRFs from blurred images, and Chen et al. [20] from a pair of sharp and blurred images. CRF estimation from multiple images was tackled before the single image estimation case. The seminal work of Debevek and Malik [11] is the first major work on the topic, where images obtained at different exposure values were used to approximate a single CRF function. Other important works are those of Mitsunaga and Nayar [21] and Mann [22]. Recently, methods obtaining the camera CRF from differently illuminated images have appeared [23]-[25]. We want to stress that our method, although using more than one image, is different in nature to the multiple image approaches just mentioned. Those methods need a set of images taken with the same settings in order to obtain a single CRF, while our method obtains as many gamma estimates as there are images. A method that, similarly to ours, obtains as many CRFs as images used is the one of Díaz and Sturm [12]; however, it relies in strong computer vision assumptions: the cameras must be geometrically calibrated and a 3D scene model is required.

Let us also remark that none of the single-image methods, neither the blind gamma estimation [14], [15], [16], nor the CRF estimation ones [10], [17], are capable of improving their performance if extra images are available.

\section{GAMMA ESTIMATION FROM MULTIPLE IMAGES OF THE SAME SCENE}

\section{A. Ratio estimation as a pre-processing for color stabilization}

Let us consider two images $\hat{I}_{1}, \hat{I}_{2}$ of the same scene, not necessarily registered, taken under two different settings of the same camera. Let us also assume that the illumination has not changed, so if $p$ is a scene point appearing in both images then it has produced in the sensor the same triplet $(R, G, B)_{p}$ in both pictures. In each of the two images the value of the pixel $p$ will most likely appear at different locations and with different pixel values, $(R, G, B)_{p 1}$ and $(R, G, B)_{p 2}$, where the number subscript represents a particular setting. Recently, Vazquez-Corral and Bertalmío [13] proposed a color stabilization method which is based on the imaging formation model presented by Bianco et al. [1]. Following this model, Vazquez-Corral and Bertalmío represent the pixel values as:

$$
\left[\begin{array}{l}
R \\
G \\
B
\end{array}\right]_{p 1}=\left(A_{1}\left[\begin{array}{l}
R \\
G \\
B
\end{array}\right]_{p}\right)^{\frac{1}{\gamma_{1}}} ; \quad\left[\begin{array}{l}
R \\
G \\
B
\end{array}\right]_{p 2}=\left(A_{2}\left[\begin{array}{l}
R \\
G \\
B
\end{array}\right]_{p}\right)^{\frac{1}{\gamma_{2}}}
$$

where $A_{1}$ and $A_{2}$ are $3 \times 3$ matrices. From here, they got the following relation

$$
\left[\begin{array}{l}
R \\
G \\
B
\end{array}\right]_{p 1}^{\gamma 1}=H\left[\begin{array}{l}
R \\
G \\
B
\end{array}\right]_{p 2}^{\gamma 2}
$$

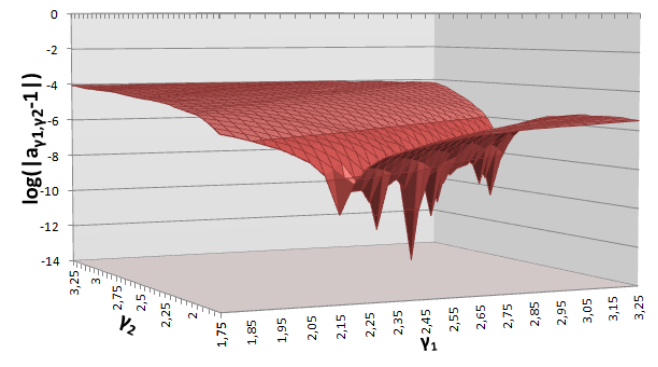

Fig. 1. $\log \left(\left|a_{\tilde{\gamma_{1}}}, \tilde{\gamma_{2}}-1\right|\right)$ depending on the different values of the gammas. We see that a set of minima is achieved for a particular ratio of gamma values: all the local minima lie on a line, corresponding to the correct ratio $\frac{\gamma_{1}}{\gamma_{2}}$.

where $H=A_{1} \cdot A_{2}^{-1}$.

This relationship holds for all pairs $p 1, p 2$ of corresponding pixels between the two images. Therefore, each pair of pixel correspondences gives us an equation, and we can find $H$ by solving a system of equations, assuming we know $\gamma_{1}$ and $\gamma_{2}$. For color stabilization it was shown in [13] that it suffices with computing the ratio $\frac{\gamma_{1}}{\gamma_{2}}$, which was done as explained below.

Let us suppose that we have obtained a set of pixel matches $\left(P_{i}, Q_{i}\right)$ between $\hat{I}_{1}$ and $\hat{I}_{2}$, i.e. $\hat{I}_{1}\left(P_{i}\right) \simeq \hat{I}_{2}\left(Q_{i}\right) \forall i$. Then, our previous Eq. 3 can be written in the form

$$
\hat{I}_{1}\left(P_{i}\right)^{\gamma_{1}}=H \hat{I}_{2}\left(Q_{i}\right)^{\gamma_{2}}, \forall i
$$

Eq. (4) tells us that for the actual $\gamma_{1}$ and $\gamma_{2}$ values there exists a matrix $H$ yielding a perfect equality. Generalizing Eq. (4) to cases where this equality is not satisfied we obtain

$$
\hat{I}_{1}\left(P_{i}\right)^{\tilde{\gamma_{1}}}=a_{\tilde{\gamma_{1}}, \tilde{\gamma_{2}}} H_{\tilde{\gamma_{1}}, \tilde{\gamma_{2}}} \hat{I}_{2}\left(Q_{i}\right)^{\tilde{\gamma_{2}}}+b_{\tilde{\gamma_{1}}, \tilde{\gamma_{2}}}
$$

where $a_{\tilde{\gamma_{1}}, \tilde{\gamma_{2}}}$ and $b_{\tilde{\gamma_{1}}, \tilde{\gamma_{2}}}$ are found by correlation between $\hat{I_{1}}\left(P_{i}\right)^{\tilde{\gamma_{1}}}$ and $H_{\tilde{\gamma_{1}}, \tilde{\gamma_{2}}} \hat{I_{2}}\left(Q_{i}\right)^{\tilde{\gamma_{2}}}$ for each pre-set value of $\tilde{\gamma_{1}}, \tilde{\gamma_{2}}$.

Therefore, to obtain the values of $\gamma_{1}$ and $\gamma_{2}$ that better approximate Eq. 4 we look for the pair $\left(\gamma_{1}, \gamma_{2}\right)$ that minimizes

$$
\left(\gamma_{1}, \gamma_{2}\right)=\arg \min _{\tilde{\gamma_{1}}, \tilde{\gamma_{2}}}\left|a_{\tilde{\gamma_{1}}}, \tilde{\gamma_{2}}-1\right|
$$

This minimization is performed via a brute-force search, i.e., all the values for a particular range of gammas are tested. Fig. 1 shows the result of this process for two images. We can see that a clear set of minima is achieved for a particular ratio of gamma values: all the local minima lie on a line, corresponding to the correct ratio $\frac{\gamma_{1}}{\gamma_{2}}$. We believe this can be explained by the fact that, for the correct values of $\tilde{\gamma}_{1}, \tilde{\gamma_{2}}$, we have $a_{\tilde{\gamma_{1}}, \tilde{\gamma_{2}}}=1$ and $b_{\tilde{\gamma_{1}}, \tilde{\gamma_{2}}}=0$, and therefore for other values $k \tilde{\gamma_{1}}, k \tilde{\gamma_{2}}$ satisfying the same ratio Eq. 5 can be approximated by Eq. 4 with only a minor change in the values for the matrix $H$, which is able to encode most of the error committed. Fig. 1 shows us that two images are enough to compute the ratio of the $\gamma$ values but might not be enough for the computation of the exact values since the real solution might not fall on the global minimum, but in a local one.

\section{B. Estimating the gamma values of multiple images}

First, let us note that the range of $\gamma$ values introduced by cameras is limited. In particular, most of the cameras embed 


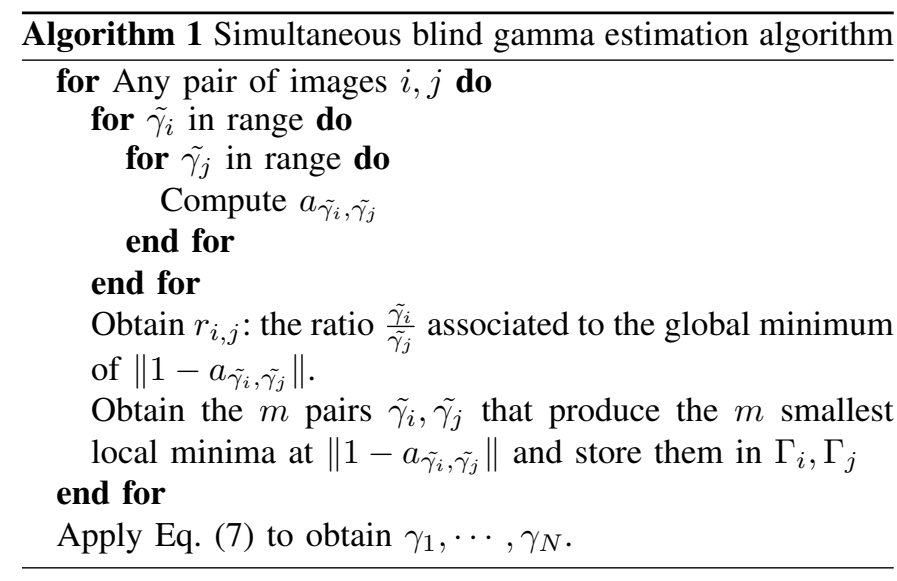

a gamma value ranging between 2 and 3. This fact, combined with the ratio estimation from the previous section, allows us to devise a new method for multiple blind gamma estimation.

Let us start by a detailed explanation of our idea. Let us suppose we have two images $I_{1}$ and $I_{2}$. From the previous section, we find the ratio between their respective gamma values: $\gamma_{1}=r_{1,2} \gamma_{2}$. As an example, let this ratio be $r_{1,2}=0.9$. In this case, $\gamma_{1}$ will take values in the interval $[2-2.7]$ as both $\gamma_{1}$ and $\gamma_{2}$ values should be in the range [2-3] and the ratio between them should be kept. Let us suppose we take a third image $I_{3}$. Following the same procedure we find the ratio relating the gamma value of this third image to $I_{1}: \gamma_{1}=r_{1,3} \gamma_{3}$. Let this ratio be $r_{1,3}=1.25$. From this second ratio, the value of $\gamma_{1}$ should be in the interval [2.5-3]. Intersecting the intervals where $\gamma_{1}$ should be, we find that $\gamma_{1} \in[2.5-2.7]$. As further images are added to the computation the possible interval where $\gamma_{1}$ is located gets further reduced, therefore helping the estimation process. This process is analogously performed for the gamma values of the other images.

Let us now detail how to automatize the procedure just explained. From Fig. 1 we see that the minimization proposed by Eq. 6 presents a set of local minima. Let us select for each comparison the set of the $m$ smaller local minima, and the ratio of the comparison, $r_{i, j}$, as the ratio between the global minimizers of Eq. 6. Let us now suppose that we have $N$ different images of the same scene under different settings: $\left\{\hat{I}_{i}\right\}_{i=1: N}$. As for each comparison we have extracted $m$ local minima, we end up with $K=(N-1) \times m$ possible values of gamma for each particular image (i.e. the number of pairs the image is involved in multiplied by the possible solutions extracted for each pair). Let us call the set of these possible solutions $\left\{\Gamma_{i}\right\}_{i=1: N}$. From this set we need to select the best possible $\left\{\gamma_{i}\right\}_{i=1: N}$ for each image. The novelty is that to this end, we define a paradigm to obtain the best gamma estimates for all images at once by searching in the possible solutions of $\Gamma_{1}, \cdots, \Gamma_{n}$. We find the values $\gamma_{1}, \cdots, \gamma_{N}$ minimizing the differences with the ratios $r_{i, j}$, which we assume have been accurately estimated

$$
\begin{aligned}
& \left(\gamma_{1}, \cdots, \gamma_{N}\right)= \\
& =\underset{\tilde{\gamma}_{k} \in \Gamma_{k}}{\arg \min } \sum_{i=1}^{N-1} \sum_{j=i+1}^{N}\left(\frac{\tilde{\gamma}_{i}}{\tilde{\gamma}_{j}}-r_{i, j}\right)^{2}+\left(\frac{\tilde{\gamma}_{j}}{\tilde{\gamma}_{i}}-\frac{1}{r_{i, j}}\right)^{2} .
\end{aligned}
$$

In this last equation we use both the ratio $r_{i, j}$ and its inverse to not prioritize errors committed in ratios smaller than 1 . An algorithmical explanation is presented in Algorithm 1. Our method takes around 6 seconds per pair comparison using a Matlab prototype on images of $900 \times 1600$ pixels, and considering gammas between 2 and 3 at 0.05 intervals. This runtime may be further reduced with an optimized C++ implementation or using a language such as CUDA, given that our algorithm is easily parallelizable.

Let us note that our method fails when both the camera settings and the scene conditions are the same for both images, since in that case we have $H=I d$ and any $\gamma_{1}=\gamma_{2}$ combination will yield the same result, therefore not obtaining any local minima. However, this is a very unrealistic situation, almost limited to the use of the same image twice, since just a minor change on the illumination, or the use of any automatic option of the camera will break this assumption.

\section{EXPERIMENTS AND RESUltS}

We have run three different experiments designed to emulate different real-world situations: (1) registered images from the same camera under different settings, (2) unregistered images from the same camera under different settings, and (3) unregistered images from different cameras and illuminations.

\section{A. Registered images from the same camera}

We captured 48 different RAW images with a Nikon D3100 camera. For each RAW image we obtained 7 different JPEG images by multiplying the RAW image by a $3 \times 3$ matrix $A$ that varies in every case and then applying gamma correction with a random $\gamma \in[2,3]$. All these computations were performed in 12 bits, emulating the camera in-processing behavior. Finally, we quantized the images to 8 bits and saved them in JPEG format. Top rows of Fig. 2 present an example of these images.

In this experiment, since the images are registered, our method considers all points as matches. We have run Eq. 7 for a different number of input images considering 3 local minima for each comparison $(m=3)$. For the sake of fair comparisons one image of the set is randomly designed as anchor and results for all the methods are computed for this image. We compare versus the well-known methods of Farid [14] and Lin et al. [10]. In the case of Lin et al. the $\gamma$ value was computed by looking for the value that best approximated the CRF obtained from the implementation of [26]. Estimates of the $\gamma$ value for all the methods were computed in the range $[2,3]$. Table I shows the results. Our method improves the others even in the case of considering just two images, and better results are achieved when using a higher number of images, reaching to an error close to only $5 \%$. Our improvement ranges between $25 \%$ in the worst case (RMS-2 images) and $70 \%$ in the best case (median-7 images). Our method performs extremely well for the median, which was proven to be the best error measure for a related problem as computational color constancy [27]. Let us recap here that our method is different from those using several images for obtaining a single CRF: in our case, every time a new image is added, also a new $\gamma$ value is estimated. Finally, we want to stress again that none of the other methods improve accuracy if more than a single image is available. 


\section{B. Unregistered images from the same camera}

We collected 10 different 'scene' sets with a single camera, a Panasonic Lumix DMC-FZ8. Each scene set is composed by a RAW image and 5 JPEG images that vary in the camera settings. The selected settings were: portrait, landscape, sunset, snow and aerial. All the images were captured without flash. From the RAW image of the scene set we create a JPEG image in a procedure that is analogous to the previous experiment, now with $\gamma \in[1.6,2.6]$. This last image is the one we are going to use as ground truth since we do know the value of $\gamma$. Middle rows of Fig. 2 present an example of these images.

We have run our method considering as matches those obtained by a SIFT procedure (using the code in [28]), and automatically discarding those that were incorrect in terms of color or image geometry. We have again used 3 local minima. In this case, the estimators were computed in the range $[1.5-2.75]$. We evaluate the methods in a slightly bigger range from that $\gamma$ so as to allow the methods to deviate from the solution in case they fail. Results are presented in Table I. We can see how our method outperforms the other two also in this scenario. Again, we want to focus the reader's attention in the median, where the improvement ranges between $12 \%$ and $48 \%$.

It is interesting as well to analyze what happens to the other 5 images that have been obtained with the different camera settings. All the images sharing the same settings should theoretically have the same $\gamma$ value. Therefore, to further evaluate our method we have computed the standard deviation of our $\gamma$ estimate between images with the same settings, and found that it is always below $10 \%$ of the $\gamma$ value.

\section{Multi-camera and illuminant variation case}

The goal of this last experiment is to check if our method can be used when not all of our original assumptions hold, i.e. when the images come from different cameras and when there is a change in the scene illumination. To this end, we captured 9 RAW scenes at midday with two different cameras: a Nikon D3100 (camera 1) and a Panasonic Lumix DMC-FZ8 (camera 2). From these 18 RAW images we created JPEG versions following the procedure of Section IV-A, with $\gamma$ values ranging between 1.6 and 2.6. We also captured at late afternoon the same 9 scenes with the second camera. In these new images, larger point of view changes were also allowed. From these new 9 RAW images we also created JPEG versions. Example images can be found in the bottom rows of Fig. 2 .

We have run our experiment twice: First, we consider the images of camera 1 and those of camera 2 at midday, and second, we consider the images of camera 1 and those of camera 2 at late afternoon. As in the previous section, image matches were computed via a SIFT descriptor and three local minima were considered. Results are presented in Table I. We can see from these numbers that our proposed approach outperforms the other two methods both in the case of same illumination and in the case of different time-of-theday illumination reaching to at least $30 \%$ improvement for the median measure. These results make us confident of the applicability of our method to images where there does not

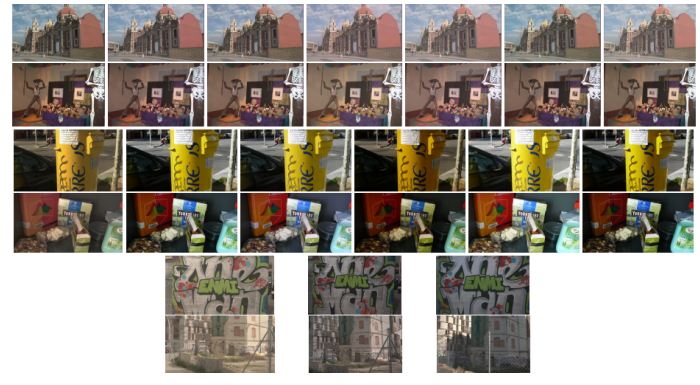

Fig. 2. Example images for the three datasets. First dataset (top 2 rows): each scene has 7 different images, varying both in the color matrix $A$ and in the gamma value $\gamma$. Second dataset (middle 2 rows): the first column shows the image created from the original RAW using a known $\gamma$ value and the other columns show camera JPEGs obtained with different scene settings. Third dataset (last 2 rows): the first column shows the camera 1 at midday, the second one the camera 2 at midday, and the third the camera 2 at evening.

TABLE I

PERCENTAGE ERROR OF THE GAMMA ESTIMATION OF $n$ IMAGES

\begin{tabular}{|c||c|c|c|}
\hline \multicolumn{1}{|c||}{ Method } & Median & Mean & RMS \\
\hline \multicolumn{1}{|c||}{ Experiment 1 } \\
\hline Farid [14] (single image) & $14.15 \%$ & $14.52 \%$ & $16.96 \%$ \\
\hline Lin et al. [10] (single image) & $15.25 \%$ & $15.90 \%$ & $17.43 \%$ \\
\hline Proposed: 2 images & $7.69 \%$ & $10.02 \%$ & $12.65 \%$ \\
\hline Proposed: 3 images & $8.64 \%$ & $9.26 \%$ & $11.19 \%$ \\
\hline Proposed: 4 images & $6.92 \%$ & $7.40 \%$ & $9.04 \%$ \\
\hline Proposed: 5 images & $5.65 \%$ & $6.93 \%$ & $8.94 \%$ \\
\hline Proposed: 6 images & $5.16 \%$ & $6.07 \%$ & $7.77 \%$ \\
\hline Proposed: 7 images & $4.32 \%$ & $5.32 \%$ & $6.76 \%$ \\
\hline Farid [14] (single image) & $23.19 \%$ & $24.36 \%$ & $24.84 \%$ \\
\hline Lin et al. [10] (single image) & $8.99 \%$ & $9.85 \%$ & $11.53 \%$ \\
\hline Proposed: 2 images & $7.62 \%$ & $9.36 \%$ & $11.04 \%$ \\
\hline Proposed: 4 images & $5.62 \%$ & $7.65 \%$ & $10.34 \%$ \\
\hline Proposed: 6 images & $4.47 \%$ & $6.17 \%$ & $8.04 \%$ \\
\hline Farid [14] (single image) & $28.88 \%$ & $27.13 \%$ & $29.62 \%$ \\
\hline Lin et al. [10] (single image) & $17.14 \%$ & $14.25 \%$ & $16.06 \%$ \\
\hline Proposed, same illum. (2 images) & $9.35 \%$ & $10.66 \%$ & $13.09 \%$ \\
\hline Proposed, different illum. (2 images) & $12.10 \%$ & $13.16 \%$ & $16.03 \%$ \\
\hline
\end{tabular}

exist any information about their capture conditions. In fact, the second setting is equivalent to the download of an internet image, where neither camera nor illumination are known.

\section{CONCLUSION}

We have presented a method for blind gamma estimation from and for multiple images. The expression "from and for" is used as we need more than a single image to obtain the gamma estimation but, at the same time, we recover as many estimates as images are used. Our method does not require any particular prior since it relies on a very simple relationship that linear images captured with different settings must accomplish. It can be used on images of the same scene where we know nothing of the cameras used (model, settings, parameter values).

Rigorous experiments were performed to evaluate the capability of our method to deal with different real life situations showing that it outperforms the state-of-the-art.

Further work will focus on improving the selection of pixel correspondences and studying possible alternatives to Eq. 7, striving for even better accuracy and robustness to changes of illumination and point of view. 


\section{REFERENCES}

[1] S. Bianco, A. Bruna, F. Naccari, and R. Schettini, "Color space transformations for digital photography exploiting information about the illuminant estimation process," J. Opt. Soc. Am. A, vol. 29, pp. 374-384, Mar 2012.

[2] M. Bertalmío, Image Processing for Cinema. Chapman \& Hall/CRC Mathematical and Computational Imaging Sciences Series, CRC Press - Taylor \& Francis, 1st ed., 2014.

[3] M. Grossberg and S. Nayar, "What is the Space of Camera Response Functions?," in IEEE Conference on Computer Vision and Pattern Recognition (CVPR), vol. II, pp. 602-609, Jun 2003.

[4] G. Sharma, ed., Digital Color Imaging Handbook, 2nd Edition. Boca Raton, FL: CRC Press, 2013.

[5] R. Ramanath, W. Snyder, Y. Yoo, and M. Drew, "Color image processing pipeline," Signal Processing Magazine, IEEE, vol. 22, pp. 34-43, Jan 2005.

[6] R. Lukac, ed., Single-Sensor Imaging: Methods and Applications for Digital Cameras. Boca Raton, FL: CRC Press, 2008.

[7] A. Gijsenij, T. Gevers, and J. van de Weijer, "Computational color constancy: Survey and experiments," IEEE Transactions on Image Processing, vol. 20, no. 9, 2011.

[8] J. Vazquez-Corral, M. Vanrell, R. Baldrich, and F. Tous, "Color constancy by category correlation.," IEEE Transactions on Image Processing, vol. 21, no. 4, pp. 1997-2007, 2012.

[9] R. Fergus, B. Singh, A. Hertzmann, S. T. Roweis, and W. T. Freeman, "Removing camera shake from a single photograph," ACM Trans. Graph., vol. 25, pp. 787-794, July 2006.

[10] S. Lin, J. Gu, S. Yamazaki, and H.-Y. Shum, "Radiometric calibration from a single image.," in CVPR (2), pp. 938-945, 2004.

[11] P. E. Debevec and J. Malik, "Recovering high dynamic range radiance maps from photographs," in Proceedings of the 24th Annual Conference on Computer Graphics and Interactive Techniques, SIGGRAPH '97, (New York, NY, USA), pp. 369-378, ACM Press/Addison-Wesley Publishing Co., 1997.

[12] M. Díaz and P. Sturm, "Radiometric Calibration using Photo Collections," in ICCP 2011 - IEEE International Conference on Computational Photography, (Pittsburgh, États-Unis), pp. 1-8, IEEE, Carnegie Mellon University, IEEE Computer Society, Apr. 2011.

[13] J. Vazquez-Corral and M. Bertalmío, "Color stabilization along time and across shots of the same scene, for one or several cameras of unknown specifications," Image Processing, IEEE Transactions on, vol. 23, pp. 4564-4575, Oct 2014.

[14] H. Farid, "Blind inverse gamma correction," Image Processing, IEEE Transactions on, vol. 10, pp. 1428-1433, Oct 2001.

[15] M. C. Stamm and K. J. R. Liu, "Blind forensics of contrast enhancement in digital images.," in ICIP, pp. 3112-3115, IEEE, 2008.

[16] G. Cao, Y. Zhao, and R. Ni, "Forensic estimation of gamma correction in digital images.," in ICIP, pp. 2097-2100, IEEE, 2010.

[17] S. Lin and L. Zhang, "Determining the radiometric response function from a single grayscale image.," in $C V P R(2)$, pp. 66-73, IEEE Computer Society, 2005.

[18] T.-T. Ng, S.-F. Chang, and M.-P. Tsui, "Using geometry invariants for camera response function estimation.," in CVPR, IEEE Computer Society, 2007.

[19] Y.-W. Tai, X. Chen, S. Kim, S. J. Kim, F. Li, J. Yang, J. Yu, Y. Matsushita, and M. S. Brown, "Nonlinear camera response functions and image deblurring: Theoretical analysis and practice," IEEE Transactions on Pattern Analysis and Machine Intelligence, vol. 35, no. 10, pp. 24982512, 2013.

[20] X. Chen, F. Li, J. Y., and J. Yu, "A theoretical analysis of camera response functions in image deblurring.," in $E C C V$ (7) (A. W. Fitzgibbon, S. Lazebnik, P. Perona, Y. Sato, and C. Schmid, eds.), vol. 7578 of Lecture Notes in Computer Science, pp. 333-346, Springer, 2012.

[21] T. Mitsunaga and S. Nayar, "Radiometric Self Calibration," in IEEE Conference on Computer Vision and Pattern Recognition (CVPR), vol. 1, pp. 374-380, Jun 1999.

[22] S. Mann, "Comparametric equations with practical applications in quantigraphic image processing.," IEEE Transactions on Image Processing, vol. 9, no. 8, pp. 1389-1406, 2000.

[23] S. J. Kim, J.-M. Frahm, and M. Pollefeys, "Radiometric calibration with illumination change for outdoor scene analysis.," in CVPR, IEEE Computer Society, 2008.

[24] B. Shi, Y. Matsushita, Y. Wei, C. Xu, and P. Tan, "Self-calibrating photometric stereo," in IEEE Conference on Computer Vision and Pattern Recognition (CVPR), 2010
[25] K. Shafique and M. Shah, "Estimation of the radiometric response functions of a color camera from differently illuminated images," in in Proceedings of the IEEE International Conference on Image Processing, pp. 24-27, 2004.

[26] J. Lalonde, "http://vision.gel.ulaval.ca/ jflalonde/software.html."

[27] S. D. Hordley and G. D. Finlayson, "Reevaluation of color constancy algorithm performance," J. Opt. Soc. Am. A, vol. 23, pp. 1008-1020, May 2006.

[28] A. Vedaldi and B. Fulkerson, "Vlfeat: An open and portable library of computer vision algorithms," in Proceedings of the international conference on Multimedia, pp. 1469-1472, ACM, 2010.

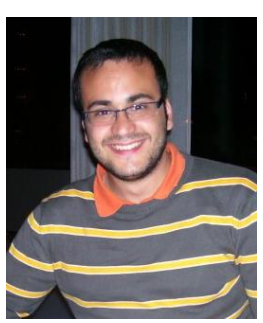

Javier Vazquez-Corral received the Ph.D. degree in computer science from the Universitat Autnoma de Barcelona (UAB), Bellaterra, Spain, in 2011. He is a Postdoctoral fellow at Universitat Pompeu Fabra (UPF) in Barcelona. His research interests are related to the use of color in image processing and computer vision problems. He is also interested in bridging the gap between color in the human brain and its use in computer-vision applications.

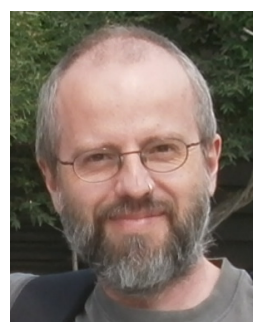

Marcelo Bertalmío received the Ph.D. degree in electrical and computer engineering from the University of Minnesota in 2001. He is an Associate Professor at University Pompeu Fabra in Barcelona, Spain. His interests are Image Processing and Computer Vision for digital cinema applications, although he prefers the (analog) films of Joseph L. Mankiewicz and Luis García Berlanga. 